\title{
SIMULTANEOUS OPTICAL TO NEAR-IR OBSERVATIONS OF BLAZARS.
}

\author{
R. FALOMO and M. BERSANELLI \\ Osservatorio Astronomico di Padova, v. Osservatorio 5, 35122 Padova, Italy \\ Istituto di Fisica Cosmica, CNR, v. Bassini 15, 20133 Milano, Italy
}

We obtained simultaneous optical spectrophotometry (4000-8000 $\AA$ ) and nearIR photometry (JHKL) for 34 blazars, with repeated observations for 21 of them. All the data were taken at ESO and cover a period of 3 years with a typical time interval of 6 months. The observations were corrected for interstellar extinction and analyzed using a uniform procedure. This allows to form a large and homogeneous data set of optical-near-IR measurements of blazars emission.

For each observation we constructed a composite spectral flux distribution (SFD) covering the range $8 \times 10^{13}-7 \times 10^{14} \mathrm{~Hz}$. We find that, with very few exceptions, the SFD of each object is well described either by a single power law $\left(f_{\nu} \propto \nu^{-\alpha}\right)$ or by a power law plus the contribution of a giant elliptical host galaxy (see Fig. 1). Our results show that, contrary to other findings, negative $\left(d \log \left(f_{\nu}\right) / d \log (\nu)<0\right)$ spectral curvatures in the optical to near-IR, when observed, can be easily ascribed to the contribution from a host galaxy and the effect of galactic extinction.

For our sample we find that the average spectral index of the non-thermal emission is $\langle\alpha\rangle=1.08 \pm 0.06$. Moreover the $\mathrm{X}$-ray selected objects exhibit a significantly (99\% c.l. for a KS test) flatter spectra than radio selected ones.

For $\sim 30 \%$ of the sources we have repeated observations. The optical and near-IR emission are strongly correlated and usually maintain the spectral index $\left(\left\langle\Delta \alpha_{\max }\right\rangle\right.$ $=0.17 \pm 0.04)$ even under substantial flux variations. No evidence of correlation between $\alpha$ and flux level is observed. A full report of these observations is given elsewhere [1].
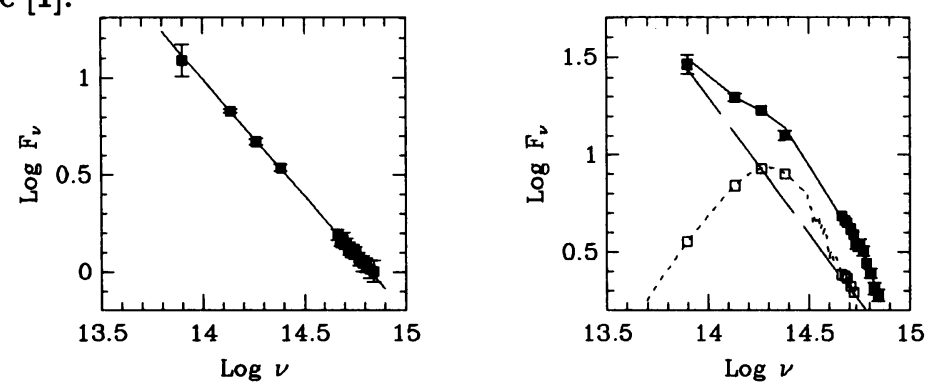

Fig. 1. Representative SFD for two objects: 0048-097 (left), and 0521-365 (right).

\section{References}

[1] Falomo R., Bersanelli M., Bouchet P., and Tanzi E.G. 1993, AJ, 106, 11

T. J.-L. Courvoisier and A. Blecha: Multi-Wavelength Continuum Emission of AGN, 321. (C) 1994 IAU. Printed in the Netherlands. 